\title{
Hyers-Ulam Stability for Quantum Equations of Euler Type
}

\author{
Douglas R. Anderson $\mathbb{D i D}^{1}$ and Masakazu Onitsuka ${ }^{2}$ \\ ${ }^{1}$ Department of Mathematics, Concordia College, Moorhead, MN 56562, USA \\ ${ }^{2}$ Okayama University of Science, Department of Applied Mathematics, Okayama 700-0005, Japan \\ Correspondence should be addressed to Douglas R. Anderson; andersod@cord.edu
}

Received 22 March 2020; Revised 5 May 2020; Accepted 8 May 2020; Published 18 May 2020

Academic Editor: Marek Galewski

Copyright (C 2020 Douglas R. Anderson and Masakazu Onitsuka. This is an open access article distributed under the Creative Commons Attribution License, which permits unrestricted use, distribution, and reproduction in any medium, provided the original work is properly cited.

\begin{abstract}
Many applications using discrete dynamics employ either $q$-difference equations or $h$-difference equations. In this work, we introduce and study the Hyers-Ulam stability (HUS) of a quantum ( $q$-difference) equation of Euler type. In particular, we show a direct connection between quantum equations of Euler type and $h$-difference equations of constant step size $h$ with constant coefficients and an arbitrary integer order. For equation orders greater than two, the $h$-difference results extend first-order and second-order results found in the literature, and the Euler-type $q$-difference results are completely novel for any order. In many cases, the best HUS constant is found.
\end{abstract}

\section{Introduction}

Recently, there has been much interest in questions of Hyers-Ulam stability for differential equations and $h$-difference equations, but little has been published specifically on $q$-difference (quantum) equations [1], in particular, on quantum equations of Euler type. In this work, we introduce a new and direct connection between Hyers-Ulam stability results for $h$-difference equations with constant coefficients, of first, second, and all higher orders, with Hyers-Ulam stability results for quantum equations of Euler type, of all integer orders, through a change of variables. First, we will connect the two types of equations and then introduce Hyers-Ulam stability.

The results in this paper connecting $h$-difference equations and $q$-difference equations of Euler type are novel. Even if we just consider the higher-order $h$-difference results independently, they extend first-order and second-order results found in $[2,3]$ to $n$th order and are not the same as the results in [4-6], where $h=1$ and different techniques are used. For a great introduction to quantum calculus, see the monograph [1], which has sections on both $q$-calculus and $h$-calculus, but does not show the nexus that we do here.

The rest of the paper will develop as follows. In Section 2, we establish the connection between $q$-difference equations of the Euler type and $h$-difference equations, via a change of variable. We then define Hyers-Ulam stability (HUS) and prove for which the parameter values the first-order $q$-difference equation of the Euler type has HUS; in the case that it does exhibit HUS, a minimum HUS constant is found. In Section 3, the Hyers-Ulam stability of second-order quantum equations of the Euler type is established from known results for $h$-difference equations. In Section 4, the stability of both higher-order quantum equations of the Euler type and higher-order $h$-difference equations with constant coefficients is proven by mathematical induction; these results are new in each context. For some cases, the best HUS constant is found. In Section 5, higher-order perturbed quantum equations of the Euler type and higher-order perturbed $h$-difference equations with constant (complex) coefficients are analyzed, and HUS with specific HUS constants is established for each setting.

\section{Connections and First-Order Stability}

Lemma 1. Assume $q>1$ and $h>0$ and set

$$
\begin{aligned}
q^{\mathbb{Z}} & =\left\{\ldots, q^{-2}, q^{-1}, 1, q, q^{2}, \ldots\right\}, \\
h \mathbb{Z} & =\{\ldots,-2 h,-h, 0, h, 2 h, \ldots\} .
\end{aligned}
$$


Let $\alpha \in \mathbb{C} \backslash\{-1 /(q-1)\}$ and $\lambda \in \mathbb{C} \backslash\{-1 / h\}$ be given, and let $I$ be the identity operator. Then, the (factored) quantum equation of the Euler type

$$
\begin{aligned}
\left(s D_{q}-\alpha I\right) y(s) & =g(s), \\
D_{q} y(s) & :=\frac{y(q s)-y(s)}{(q-1) s},
\end{aligned}
$$

has a solution $y$ for $s \in q^{\mathbb{Z}}$ if and only if the (factored) $h$-difference equation

$$
\begin{aligned}
\left(\Delta_{h}-\lambda I\right) x(t) & =f(t), \\
\Delta_{h} x(t) & :=\frac{x(t+h)-x(t)}{h},
\end{aligned}
$$

has a solution $x$ for $t \in h \mathbb{Z}$, where

$$
\begin{aligned}
& t=h \log _{q} s \text { and } \lambda=\frac{\alpha(q-1)}{h}, \quad \text { equivalently } \\
& s=q^{t / h} \text { and } \alpha=\frac{\lambda h}{q-1},
\end{aligned}
$$

is a change of variables between $s$ and $\alpha$ to $t$ and $\lambda$, while

$$
\begin{gathered}
x(t)=\frac{h}{q-1} y\left(q^{t / h}\right) \& f(t)=g\left(q^{t / h}\right), \quad \text { i.e. } \\
y(s)=\frac{q-1}{h} x\left(h \log _{q} s\right) \& g(s)=f\left(h \log _{q} s\right),
\end{gathered}
$$

is a change of functions between the variables.

Proof. Let $y$ be a solution of $\left(s D_{q}-\alpha I\right) y(s)=g(s)$ for $\alpha \in \mathbb{C} \backslash\{-1 /(q-1)\}$. Then, the change of variables (4) converts this equation to

$$
\frac{h}{q-1}\left(\frac{y\left(q^{(t+h) / h}\right)-y\left(q^{t / h}\right)}{h}\right)-\frac{h \lambda}{q-1} y\left(q^{t / h}\right)=g\left(q^{t / h}\right),
$$

where $\lambda \in \mathbb{C} \backslash\{-1 / h\}$. Now, make the change of functions (5). Then, the function $x$ is a solution of $\left(\Delta_{h}-\lambda I\right) x(t)=f(t)$. Using (4) and (5), this process is reversible, yielding the converse.

Definition 1. Assume $q>1$ and $\alpha \in \mathbb{C} \backslash\{-1 /(q-1)\}$. The Euler-type quantum equation

$$
\left(s D_{q}-\alpha I\right) y(s)=0,
$$

has Hyers-Ulam stability (HUS) if and only if there exists a constant $K>0$ with the following property. For an arbitrary $\varepsilon>0$, if a function $\psi: q^{\mathbb{Z}} \longrightarrow \mathbb{C}$ satisfies

$$
\left|\left(s D_{q}-\alpha I\right) \psi(s)\right| \leq \varepsilon,
$$

for all $s \in q^{\mathbb{Z}}$, then there exists a solution $y: q^{\mathbb{Z}} \longrightarrow \mathbb{C}$ of (7) such that

$$
|\psi(s)-y(s)| \leq K \varepsilon
$$

for all $s \in q^{\mathbb{Z}}$. Such a constant $K$ is called an HUS constant for (7) on $q^{\mathbb{Z}}$.

Remark 1. If, given an arbitrary $\varepsilon>0$, there exists a function $\psi$ such that (8) holds for $s \in q^{\mathbb{Z}}$, then

$$
\left|\left(\Delta_{h}-\lambda I\right) \phi(t)\right| \leq \varepsilon, \quad t \in h \mathbb{Z},
$$

holds as well, where we have used the change of variable (4) and, similar to (5), the change of function

$$
\phi(t)=\frac{h}{q-1} \psi\left(q^{t / h}\right),
$$

to rewrite (8) as (10).

Lemma 2. Assume $q>1$ and $\alpha \in \mathbb{C} \backslash\{-1 /(q-1)\}$. If $|\alpha(q-1)+1|=1$, then the quantum equation of the Euler type given in (7) is not Hyers-Ulam stable.

Proof. Assume $h>0$ and $\lambda \in \mathbb{C} \backslash\{-1 / h\}$. Using ([2], Remark $2.1)$, if $|\lambda h+1|=1$, then the $h$-difference equation,

$$
\left(\Delta_{h}-\lambda I\right) x(t)=0
$$

is not Hyers-Ulam stable. By the change of variables (4) and change of functions (5) and (11), which connect (7) to (12) and (8) to (10), the result follows.

Remark 2. Throughout the rest of the paper, let

$$
K(\tau, \theta):=\frac{\tau}{|1-| \theta \tau+1||},
$$

for $|\theta \tau+1| \neq 1$

Theorem 1. If $\alpha \in \mathbb{C} \backslash\{-1 /(q-1)\}$ with $|\alpha(q-1)+1| \neq 1$, then (7) has Hyers-Ulam stability with minimum HUS constant $K(q-1, \alpha)=(q-1) /(|1-| \alpha(q-1)+1||)$ on $q^{\mathbb{Z}}$.

Proof. Using ([2], Theorem 2.6), if $\lambda \in \mathbb{C} \backslash\{-1 / h\}$ with $|\lambda h+1| \neq 1$, then (12) has Hyers-Ulam stability with minimum HUS constant $K(h, \lambda)=h /|1-| \lambda h+1||$ on $h \mathbb{Z}$, for $K(\cdot, \cdot)$ given in (13). Let an arbitrary $\varepsilon>0$ be given. Suppose $\phi$ satisfies (10). By ([2], Theorem 2.5) there exists a solution $x$ of (12) such that

$$
|\phi(t)-x(t)| \leq \varepsilon K(h, \lambda), \quad t \in h \mathbb{Z},
$$

making the change using (4) and (11), we have

$$
\left|\frac{h}{q-1} \psi(s)-\frac{h}{q-1} y(s)\right| \leq \frac{h \varepsilon}{|1-| \alpha(q-1)+1||}, \quad s \in q^{\mathbb{Z}},
$$

so that

$$
|\psi(s)-y(s)| \leq \frac{(q-1) \varepsilon}{|1-| \alpha(q-1)+1||}=\varepsilon K(q-1, \alpha), \quad s \in q^{\mathbb{Z}},
$$

where $y$ is a solution of (7). Now from ([2], Lemma 2.3), we know there exists a specific function: 


$$
\phi(t):=c(\lambda h+1)^{t / h}+\left(\frac{\varepsilon h}{\gamma-(\lambda h+1)}\right) \gamma^{t / h}
$$

for $\gamma:=(\lambda h+1) /|\lambda h+1|$ that satisfies (10); then, $|\gamma|=1$ and $\gamma-(\lambda h+1) \neq 0$, whereby it is proven that the minimum HUS constant for $(12)$ is at least $K(h, \lambda)$, for $K(\cdot, \cdot)$ given in (13). By the change of variable (4) and the change of function (5), which connect (7) to (12) and (8) to (10) via (11), there exists a specific function $\psi$ satisfying (8), whereby the minimum HUS constant for (7) is thus $K(q-1, \alpha)$, and the result follows.

\section{Second-Order Quantum Equations of Euler Type}

Let $q>1, h>0$, and $\alpha_{j} \in \mathbb{C} \backslash\{-1 /(q-1)\}$ be given for $j \in\{1,2\}$.

Now, consider the second-order quantum equation of the Euler type, written in the factored operator form as

$$
\begin{aligned}
\left(s D_{q}-\alpha_{2} I\right)\left(s D_{q}-\alpha_{1} I\right) y(s) & =0, \\
D_{q} y(s) & :=\frac{y(q s)-y(s)}{(q-1) s} .
\end{aligned}
$$

Definition 2. The second-order Euler-type quantum equation (18) has Hyers-Ulam stability (HUS) if and only if there exists a constant $K>0$ with the following property: For an arbitrary $\varepsilon>0$, if a function $\psi: q^{\mathbb{Z}} \longrightarrow \mathbb{C}$ satisfies

$$
\left|\left(s D_{q}-\alpha_{2} I\right)\left(s D_{q}-\alpha_{1} I\right) \psi(s)\right| \leq \varepsilon,
$$

for all $s \in q^{\mathbb{Z}}$, then there exists a solution $y: q^{\mathbb{Z}} \longrightarrow \mathbb{C}$ of (18) such that

$$
|\psi(s)-y(s)| \leq K \varepsilon,
$$

for all $s \in q^{\mathbb{Z}}$. Such a constant $K$ is called an HUS constant for (18) on $q^{\mathbb{Z}}$.

Theorem 2. If $\alpha_{j} \in \mathbb{C} \backslash\{-1 /(q-1)\}$ with $\left|\alpha_{j}(q-1)+1\right| \neq 1$ for $j \in\{1,2\}$, then the second-order quantum equation of Euler type (18) has Hyers-Ulam stability with an HUS constant of

$$
K\left(q-1, \alpha_{1}\right) K\left(q-1, \alpha_{2}\right)=\frac{(q-1)^{2}}{|1-| \alpha_{1}(q-1)+1|| \cdot|1-| \alpha_{2}(q-1)+1||},
$$

on $q^{\mathbb{Z}}$

Proof. Let

$$
Y_{1}(s):=\left(s D_{q}-\alpha_{1} I\right) y(s)
$$

As a result, (18) implies that $\left(s D_{q}-\alpha_{2} I\right) Y_{1}(s)=0$ so that

$$
\begin{aligned}
\left(\Delta_{h}-\lambda_{2} I\right) X_{1}(t) & =0, \\
\Delta_{h} X_{1}(t) & :=\frac{X_{1}(t+h)-X_{1}(t)}{h},
\end{aligned}
$$

where we employ the change of variables

$$
\begin{aligned}
\lambda_{j} & =\frac{\alpha_{j}(q-1)}{h}, \\
t & =h \log _{q} s, \\
X_{1}(t) & :=\frac{h}{q-1} Y_{1}\left(q^{t / h}\right) .
\end{aligned}
$$

Then, we have

$$
X_{1}(t)=\left(s D_{q}-\alpha_{1} I\right)\left(\frac{h}{q-1} y(s)\right)
$$

Let

$$
x(t):=\left(\frac{h}{q-1}\right)^{2} y\left(q^{t / h}\right)
$$

Note that as $\alpha_{j} \neq-1 /(q-1)$, we have $\lambda_{j} \neq-1 / h$, for $j=\{1,2\}$. Moreover, $\left|\alpha_{j}(q-1)+1\right| \neq 1$ for $j \in\{1,2\}$ implies that $\left|\lambda_{j} h+1\right| \neq 1$ for $j \in\{1,2\}$. Consequently,

$$
X_{1}(t)=\left(\Delta_{h}-\lambda_{1} I\right) x(t),
$$

and this implies

$$
\begin{aligned}
0 & =\left(\Delta_{h}-\lambda_{2} I\right) X_{1}(t) \\
& =\left(\Delta_{h}-\lambda_{2} I\right)\left(\Delta_{h}-\lambda_{1} I\right) x(t) \\
& =\Delta_{h}^{2} x(t)-\left(\lambda_{1}+\lambda_{2}\right) \Delta_{h} x(t)+\lambda_{1} \lambda_{2} x(t) .
\end{aligned}
$$

Take

$$
\begin{aligned}
& \alpha=-\left(\lambda_{1}+\lambda_{2}\right), \\
& \beta=\lambda_{1} \lambda_{2},
\end{aligned}
$$

to match the notation used in [2], Theorem 3.4.

By [2], Theorem 3.4, the second-order $h$-difference equation (28) has HUS, with an HUS constant

$$
\begin{aligned}
& K\left(h, \frac{-\alpha+\sqrt{\alpha^{2}-4 \beta}}{2}\right) K\left(h, \frac{-\alpha-\sqrt{\alpha^{2}-4 \beta}}{2}\right) \\
& =K\left(h, \lambda_{1}\right) K\left(h, \lambda_{2}\right),
\end{aligned}
$$

on $h \mathbb{Z}$, where $K(\cdot, \cdot)$ is the constant expressed in (13). Given an arbitrary $\varepsilon>0$, suppose there exists a function $\psi$ such that

$$
\left|\left(s D_{q}-\alpha_{2} I\right)\left(s D_{q}-\alpha_{1} I\right) \psi(s)\right| \leq \varepsilon, \quad s \in q^{\mathbb{Z}} .
$$

Then, letting 


$$
\begin{aligned}
\phi(t) & :=\left(\frac{h}{q-1}\right)^{2} \psi\left(q^{t / h}\right), \\
t & =h \log _{q} s, \\
\lambda_{j} & =\frac{\alpha_{j}(q-1)}{h},
\end{aligned}
$$

for $j \in\{1,2\}$, we have

$$
\left|\left(\Delta_{h}-\lambda_{2} I\right)\left(\Delta_{h}-\lambda_{1} I\right) \phi(t)\right| \leq \varepsilon, \quad t \in h \mathbb{Z} .
$$

Therefore, by [2], Theorem 3.4, there exists a solution $x$ of (28) such that

$$
\begin{aligned}
|\phi(t)-x(t)| & \leq \varepsilon K\left(h, \frac{-\alpha+\sqrt{\alpha^{2}-4 \beta}}{2}\right) K\left(h, \frac{-\alpha-\sqrt{\alpha^{2}-4 \beta}}{2}\right) \\
& =K\left(h, \lambda_{1}\right) K\left(h, \lambda_{2}\right),
\end{aligned}
$$

which implies that

$$
\begin{aligned}
\left|\left(\frac{h}{q-1}\right)^{2} \psi\left(q^{t / h}\right)-\left(\frac{h}{q-1}\right)^{2} y\left(q^{t / h}\right)\right| & \leq \varepsilon \frac{h}{|1-| \lambda_{1} h+1||} \frac{h}{|1-| \lambda_{2} h+1||}, \\
\left|\psi\left(q^{t / h}\right)-y\left(q^{t / h}\right)\right| & \leq \varepsilon \frac{(q-1)}{|1-| \alpha_{1}(q-1)+1||} \frac{(q-1)}{|1-| \alpha_{2}(q-1)+1||},
\end{aligned}
$$

using (26). It follows that

$$
\begin{aligned}
& \frac{(q-1)}{|1-| \alpha_{1}(q-1)+1||} \frac{(q-1)}{|1-| \alpha_{2}(q-1)+1||} \\
& =K\left(q-1, \alpha_{1}\right) K\left(q-1, \alpha_{2}\right),
\end{aligned}
$$

is an HUS constant for (18), for $K(\cdot, \cdot)$ given in (13).

Corollary 1. Assume $\alpha_{j} \in \mathbb{C} \backslash\{-1 /(q-1)\}$ with $\mid \alpha_{j}(q-1)+$ $1 \mid \neq 1$ for $j \in\{1,2\}$.

(i) If $\alpha_{j}(q-1)+1 \in(0,1) \cup(1, \infty)$ for $j \in\{1,2\}$, then the second-order quantum equation of Euler type (18) has Hyers-Ulam stability with minimum HUS constant

$$
K=\frac{1}{\left|\alpha_{1} \alpha_{2}\right|}
$$

on $q^{\mathbb{Z}}$.

(ii) If $\alpha_{j}(q-1)+1 \in(-\infty,-1) \cup(-1,0)$ for $j \in\{1,2\}$, then the second-order quantum equation of Euler type

(18) has Hyers-Ulam stability with minimum HUS constant

$$
K=\frac{(q-1)^{2}}{\left|\left(\alpha_{1}(q-1)+2\right)\left(\alpha_{2}(q-1)+2\right)\right|},
$$

on $q^{\mathbb{Z}}$.

Proof. Assume $\alpha_{j} \in \mathbb{C} \backslash\{-1 /(q-1)\}$ with $\left|\alpha_{j}(q-1)+1\right| \neq 1$ for $j \in\{1,2\}$.

(i) If $\alpha_{j}(q-1)+1 \in(0,1) \cup(1, \infty)$ for $j \in\{1,2\}$, then

$$
K\left(q-1, \alpha_{1}\right) K\left(q-1, \alpha_{2}\right)=\frac{1}{\left|\alpha_{1} \alpha_{2}\right|},
$$

through simplification so that this constant is an HUS constant for (18) on $q^{\mathbb{Z}}$. Invoking [2], Theorem 3.4 (i) or [3], Corollary 3.1 and the change of variables to the corresponding $h$-difference equation, the constant

$$
K\left(h, \lambda_{1}\right) K\left(h, \lambda_{2}\right)=\frac{1}{\left|\lambda_{1} \lambda_{2}\right|},
$$

is the minimum HUS constant for the second-order $h$-difference equation (28) on $h \mathbb{Z}$. The result follows on $q^{\mathbb{Z}}$ after a change of variables back.

(ii) If $\alpha_{j}(q-1)+1 \in(-\infty,-1) \cup(-1,0)$ for $j \in\{1,2\}$, then

$$
K\left(q-1, \alpha_{1}\right) K\left(q-1, \alpha_{2}\right)=\frac{(q-1)^{2}}{\left|\left(\alpha_{1}(q-1)+2\right)\left(\alpha_{2}(q-1)+2\right)\right|},
$$

again through simplification of the expression, making this constant an HUS constant for (18) on $q^{\mathbb{Z}}$. Referring to [2], Theorem 3.4 (iii) and proceeding as in case (i) of this proof, and the result follows for (18) on $q^{\mathbb{Z}}$.

\section{Higher-Order Quantum Equations of Euler Type}

In this section, we extend the results in the previous two sections to higher-order quantum equations of the Euler type.

Let $q>1, h>0, \alpha_{j} \in \mathbb{C} \backslash\{-1 /(q-1)\}$, and $\lambda_{j} \in \mathbb{C} \backslash\{-1 / h\}$ be given for $j \in\{1,2, \ldots, n\}$. In this section, we consider the 
$n$ th-order quantum equation of the Euler type given in factored operator form by

$$
\begin{aligned}
& \left(s D_{q}-\alpha_{n} I\right)\left(s D_{q}-\alpha_{n-1} I\right) \cdots\left(s D_{q}-\alpha_{1} I\right) y(s)=0, \\
D_{q} y(s):= & \frac{y(q s)-y(s)}{(q-1) s} .
\end{aligned}
$$

Definition 3. The higher-order Euler-type quantum equation (42) has Hyers-Ulam stability (HUS) if and only if there exists a constant $K>0$ with the following property. For an arbitrary $\varepsilon>0$, if a function $\psi: q^{\mathbb{Z}} \longrightarrow \mathbb{C}$ satisfies

$$
\left|\left(s D_{q}-\alpha_{n} I\right)\left(s D_{q}-\alpha_{n-1} I\right) \cdots\left(s D_{q}-\alpha_{1} I\right) \psi(s)\right| \leq \varepsilon,
$$

for all $s \in q^{\mathbb{Z}}$, then there exists a solution $y: q^{\mathbb{Z}} \longrightarrow \mathbb{C}$ of (42) such that

$$
|\psi(s)-y(s)| \leq K \varepsilon,
$$

for all $s \in q^{\mathbb{Z}}$. Such a constant $K$ is called an HUS constant for (42) on $q^{\mathbb{Z}}$.

Theorem 3. If $\alpha_{j} \in \mathbb{C} \backslash\{-1 /(q-1)\}$ with $\left|\alpha_{j}(q-1)+1\right| \neq 1$ for $j \in\{1,2, \ldots, n\}$, then the higher-order quantum equation of Euler type (42) has Hyers-Ulam stability with an HUS constant of

$$
\prod_{j=1}^{n} K\left(q-1, \alpha_{j}\right)=\frac{(q-1)^{n}}{\prod_{j=1}^{n}|1-| \alpha_{j}(q-1)+1||},
$$

on $q^{\mathbb{Z}}$, where $K(\cdot, \cdot)$ is given in (13).

Proof. We proceed by mathematical induction on $n \in \mathbb{N}$. For $n=1$, equation (42) is simply (7) so that by Theorem 1, (7) has Hyers-Ulam stability with minimum HUS constant

$$
K\left(q-1, \alpha_{1}\right)=\frac{q-1}{|1-| \alpha_{1}(q-1)+1||},
$$

on $q^{\mathbb{Z}}$.

Let $n=2$. For an arbitrary $\varepsilon>0$, suppose there exists a function $\psi: q^{\mathbb{Z}} \longrightarrow \mathbb{C}$ that satisfies

$$
\left|\left(s D_{q}-\alpha_{2} I\right)\left(s D_{q}-\alpha_{1} I\right) \psi(s)\right| \leq \varepsilon,
$$

for all $s \in q^{\mathbb{Z}}$. If we let

$$
\left(s D_{q}-\alpha_{1} I\right) \psi(s)=\Psi_{2}(s),
$$

then

$$
\left|\left(s D_{q}-\alpha_{2} I\right) \Psi_{2}(s)\right| \leq \varepsilon,
$$

for all $s \in q^{\mathbb{Z}}$. Therefore, Hyers-Ulam stability for the firstorder equation implies there exists a solution $y_{2}$ of $\left(s D_{q}-\right.$ $\left.\alpha_{2} I\right) y_{2}(s)=0$ such that

$$
\left|\Psi_{2}(s)-y_{2}(s)\right| \leq \varepsilon K\left(q-1, \alpha_{2}\right) .
$$

Let $Y_{2}$ solve the equation:

$$
\left(s D_{q}-\alpha_{1} I\right) Y_{2}(s)=y_{2}(s) .
$$

This is possible by converting the equation using Lemma 1 to the corresponding $h$-difference equation and using the variation of parameters formula and then converting back. In (50), substitute for $\Psi_{2}$ using (48) and for $y_{2}$ using (51). Then, we can rewrite (50) as

$$
\left|\left(s D_{q}-\alpha_{1} I\right) \psi(s)-\left(s D_{q}-\alpha_{1} I\right) Y_{2}(s)\right| \leq \varepsilon K\left(q-1, \alpha_{2}\right),
$$

so that

$$
\left|\left(s D_{q}-\alpha_{1} I\right)\left(\psi-Y_{2}\right)(s)\right| \leq \varepsilon K\left(q-1, \alpha_{2}\right)=\varepsilon^{\prime} .
$$

Again, Hyers-Ulam stability for the first-order equation implies there exists a solution $y_{0}$ of (7) such that

$$
\left|\left(\psi-Y_{2}\right)(s)-y_{0}(s)\right| \leq \varepsilon^{\prime} K\left(q-1, \alpha_{1}\right),
$$

which implies that

$$
\left|\psi(s)-\left(Y_{2}+y_{0}\right)(s)\right| \leq \varepsilon K\left(q-1, \alpha_{1}\right) K\left(q-1, \alpha_{2}\right) .
$$

Note that

$$
\begin{aligned}
& \left(s D_{q}-\alpha_{2} I\right)\left(s D_{q}-\alpha_{1} I\right)\left(Y_{2}+y_{0}\right)(s) \\
& =\left(s D_{q}-\alpha_{2} I\right)\left[\left(s D_{q}-\alpha_{1} I\right) Y_{2}(s)+\left(s D_{q}-\alpha_{1} I\right) y_{0}(s)\right] \\
& =\left(s D_{q}-\alpha_{2} I\right)\left[y_{2}(s)+0\right] \\
& =\left(s D_{q}-\alpha_{2} I\right) y_{2}(s) \\
& =0,
\end{aligned}
$$

making $\left(Y_{2}+y_{0}\right)$ a solution of (42) with $n=2$. By Definition 3 , with $n=2$, equation (42) has Hyers-Ulam stability with HUS constant $K\left(q-1, \alpha_{1}\right) K\left(q-1, \alpha_{2}\right)$.

Let $n=k$ for some $k \in \mathbb{N}$. Without loss of generality, write (42) with $n=k$ as

$$
\left(s D_{q}-\alpha_{k+1} I\right)\left(s D_{q}-\alpha_{k} I\right) \cdots\left(s D_{q}-\alpha_{2} I\right) y(s)=0,
$$

where we have reindexed parameters as necessary and make the induction assumption that this equation has Hyers-Ulam stability with HUS constant

$$
\prod_{j=2}^{k+1} K\left(q-1, \alpha_{j}\right)=\prod_{j=2}^{k+1} \frac{(q-1)}{|1-| \alpha_{j}(q-1)+1||},
$$

on $q^{\mathbb{Z}}$.

Now, consider (42) with $n=k+1$, namely,

$$
\left(s D_{q}-\alpha_{k+1} I\right)\left(s D_{q}-\alpha_{k} I\right) \cdots\left(s D_{q}-\alpha_{1} I\right) y(s)=0 .
$$

For an arbitrary $\varepsilon>0$, suppose there exists a function $\psi: q^{\mathbb{Z}} \longrightarrow \mathbb{C}$ that satisfies

$$
\left|\left(s D_{q}-\alpha_{k+1} I\right)\left(s D_{q}-\alpha_{k} I\right) \cdots\left(s D_{q}-\alpha_{1} I\right) \psi(s)\right| \leq \varepsilon,
$$

for all $s \in q^{\mathbb{Z}}$. If we let

$$
\left(s D_{q}-\alpha_{1} I\right) \psi(s)=\Psi_{k+1}(s),
$$


then

$$
\left|\left(s D_{q}-\alpha_{k+1} I\right)\left(s D_{q}-\alpha_{k} I\right) \cdots\left(s D_{q}-\alpha_{2} I\right) \Psi_{k+1}(s)\right| \leq \varepsilon,
$$

for all $s \in q^{\mathbb{Z}}$. Therefore, by the induction assumption for $n=k$, Hyers-Ulam stability for that equation implies there exists a solution $y_{k+1}$ of $\left(s D_{q}-\alpha_{k+1} I\right)\left(s D_{q}-\alpha_{k} I\right) \cdots\left(s D_{q}-\right.$ $\left.\alpha_{2} I\right) y_{k+1}(s)=0$ such that

$$
\left|\Psi_{k+1}(s)-y_{k+1}(s)\right| \leq \varepsilon \prod_{j=2}^{k+1} K\left(q-1, \alpha_{j}\right) .
$$

Let $Y_{k+1}$ solve the equation

$$
\left(s D_{q}-\alpha_{1} I\right) Y_{k+1}(s)=y_{k+1}(s) .
$$

Using this result, we can rewrite (63) as

$$
\left|\left(s D_{q}-\alpha_{1} I\right) \psi(s)-\left(s D_{q}-\alpha_{1} I\right) Y_{k+1}(s)\right| \leq \varepsilon \prod_{j=2}^{k+1} K\left(q-1, \alpha_{j}\right),
$$

so that

$$
\left|\left(s D_{q}-\alpha_{1} I\right)\left(\psi-Y_{k+1}\right)(s)\right| \leq \varepsilon \prod_{j=2}^{k+1} K\left(q-1, \alpha_{j}\right)=\varepsilon^{\prime} .
$$

Again, Hyers-Ulam stability for the first-order equation implies there exists a solution $y_{0}$ of (7) such that

$$
\left|\left(\psi-Y_{k+1}\right)(s)-y_{0}(s)\right| \leq \varepsilon^{\prime} K\left(q-1, \alpha_{1}\right),
$$

which implies that

$$
\left|\psi(s)-\left(Y_{k+1}+y_{0}\right)(s)\right| \leq \varepsilon \prod_{j=1}^{k+1} K\left(q-1, \alpha_{j}\right) .
$$

Note that

$$
\begin{aligned}
& \left(s D_{q}-\alpha_{k+1} I\right) \cdots\left(s D_{q}-\alpha_{2} I\right)\left(s D_{q}-\alpha_{1} I\right)\left(Y_{k+1}+y_{0}\right)(s) \\
= & \left(s D_{q}-\alpha_{k+1} I\right) \cdots\left(s D_{q}-\alpha_{2} I\right)\left[\left(s D_{q}-\alpha_{1} I\right) Y_{k+1}(s)\right. \\
& \left.+\left(s D_{q}-\alpha_{1} I\right) y_{0}(s)\right] \\
= & \left(s D_{q}-\alpha_{k+1} I\right) \cdots\left(s D_{q}-\alpha_{2} I\right)\left[y_{k+1}(s)+0\right] \\
= & \left(s D_{q}-\alpha_{k+1} I\right) \cdots\left(s D_{q}-\alpha_{2} I\right) y_{k+1}(s) \\
= & 0
\end{aligned}
$$

by the choice of $y_{k+1}$, making $\left(Y_{k+1}+y_{0}\right)$ a solution of (42) with $n=k+1$. By Definition 3 with $n=k+1$, equation (42) has Hyers-Ulam stability with HUS constant $\prod_{j=1}^{k+1}$ $K\left(q-1, \alpha_{j}\right)$. Consequently, by the principle of mathematical induction, the overall result holds.

We now use Theorem 3 and the connection between $q$-difference equations of Euler type and $h$-difference equations with constant coefficients articulated earlier, to extend known results about (28) to general higher-order equations.
Corollary 2. Let $h>0$ be given. If $\lambda_{j} \in \mathbb{C} \backslash\{-1 / h\}$ with $\mid \lambda_{j} h+$ $1 \mid \neq 1$ for $j \in\{1,2, \ldots, n\}$, then the higher-order h-difference equation with constant coefficients in factored form given by

$$
\left(\Delta_{h}-\lambda_{n} I\right)\left(\Delta_{h}-\lambda_{n-1} I\right) \cdots\left(\Delta_{h}-\lambda_{1} I\right) x(t)=0,
$$

has Hyers-Ulam stability with an HUS constant of

$$
\prod_{j=1}^{n} K\left(h, \lambda_{j}\right)=\frac{h^{n}}{\prod_{j=1}^{n}|1-| \lambda_{j} h+1||}
$$

on $h \mathbb{Z}$, where $K(\cdot, \cdot)$ is given in (13).

Lemma 3. Assume $\alpha_{j} \in \mathbb{C} \backslash\{-1 /(q-1)\}$ with $\mid \alpha_{j}(q-1)+$ $1 \mid \neq 1$ for $j \in\{1,2, \ldots, n\}$. Then the following hold:

(i) If $\alpha_{1}, \alpha_{2}, \cdots, \alpha_{n}$ are distinct, then the general solution to (42) is given by

$$
y(s)=\sum_{j=1}^{n} a_{j}\left(\alpha_{j}(q-1)+1\right)^{\log _{q} s},
$$

where $a_{j}$ are arbitrary constants.

(ii) If $\alpha_{1}, \alpha_{2}, \cdots, \alpha_{r}$ are distinct with multiplicities $m_{1}, m_{2}$, $\cdots, m_{r}$ with $\sum_{j=1}^{r} m_{j}=n$, respectively, then the general solution to

$$
\left(s D_{q}-\alpha_{r} I\right)^{m_{r}}\left(s D_{q}-\alpha_{r-1} I\right)^{m_{r-1}} \cdots\left(s D_{q}-\alpha_{1} I\right)^{m_{1}} y(s)=0,
$$

is given by

$$
\begin{aligned}
y(s)= & \sum_{j=1}^{r}\left(\alpha_{j}(q-1)+1\right)^{\log _{q} s}\left[b_{j 0}+b_{j 1} \log _{q} s\right. \\
& \left.+b_{j 2}\left(\log _{q} s\right)^{2}+\cdots+b_{j m_{j}-1}\left(\log _{q} s\right)^{m_{j}-1}\right],
\end{aligned}
$$

where $b_{j i} \quad\left(i \in\left\{0,1,2, \ldots, m_{j}-1\right\}\right)$ are arbitrary constants.

Proof. Let $q>1$ and $h>0$, and $\alpha_{i} \in \mathbb{C} \backslash\{-1 /(q-1)\}$ with $\left|\alpha_{i}(q-1)+1\right| \neq 1$ for $i \in\{1,2, \ldots, n\}$. We now consider the change of variables and functions:

$$
\begin{aligned}
t & =h \log _{q} s, \\
\lambda_{j} & =\frac{\alpha_{j}(q-1)}{h}, \\
x(t) & =\frac{h}{q-1} y\left(q^{t / h}\right)=\frac{h}{q-1} y(s),
\end{aligned}
$$

for $j \in\{1,2, \ldots, n\}$. Then, the quantum equation of Euler type (42) has a solution $y$ for $s \in q^{\mathbb{Z}}$ if and only if the $h$-difference equation (70) has a solution $x$ for $t \in h \mathbb{Z}$ by using the same argument as in the proof of Lemma 1.

First, we will prove case (i). Suppose that $\alpha_{j}(j \in$ $\{1,2, \ldots, n\})$ are distinct. Since 


$$
\begin{aligned}
\Delta_{h}\left[\left(\lambda_{j} h+1\right)^{t / h}\right] & =\frac{1}{h}\left[\left(\lambda_{j} h+1\right)^{(t+h) / h}-\left(\lambda_{j} h+1\right)^{t / h}\right] \\
& =\lambda_{j}\left(\lambda_{j} h+1\right)^{t / h},
\end{aligned}
$$

holds, we have

$$
\left(\Delta_{h}-\lambda_{j} I\right) x_{j}(t)=0,
$$

where $x_{j}(t)=\left(\lambda_{j} h+1\right)^{t / h}$. That is, $x_{j}(t)(j \in\{1,2, \ldots, n\})$ are solutions to (70). Since $\alpha_{j}(j \in\{1,2, \ldots, n\})$ are distinct, $\lambda_{j}(j \in\{1,2, \ldots, n\})$ are also distinct. Consequently, the general solution to (70) is given by

$$
x(t)=\sum_{j=1}^{n} c_{j} x_{j}(t)=\sum_{j=1}^{n} c_{j}\left(\lambda_{j} h+1\right)^{t / h}
$$

where $c_{j}$ are arbitrary constants. Using connect (75), we can find the general solution

$$
\begin{aligned}
y(s) & =\sum_{j=1}^{n} \frac{(q-1) c_{j}}{h}\left(\alpha_{j}(q-1)+1\right)^{\log _{q} s} \\
& =\sum_{j=1}^{n} a_{j}\left(\alpha_{j}(q-1)+1\right)^{\log _{q} s}
\end{aligned}
$$

of (42).

Next, we prove case (ii). Suppose that $\alpha_{j}$ $(j \in\{1,2, \ldots, r\})$ are distinct with multiplicities $m_{j}$ $(j \in\{1,2, \ldots, r\})$ with $\sum_{j=1}^{r} m_{j}=n$, respectively. From connect (75), we see that $\lambda_{j}(j \in\{1,2, \ldots, r\})$ are also distinct with multiplicities $m_{j}(j \in\{1,2, \ldots, r\})$ with $\sum_{j=1}^{r} m_{j}=n$, respectively. By [7], Corollary 2.24, we can know that

$$
x(t)=\sum_{j=1}^{r}\left(\lambda_{j} h+1\right)^{t / h}\left[d_{j 0}+d_{j 1} t+d_{j 2} t^{2}+\cdots+d_{j m_{j}-1} t^{m_{j}-1}\right],
$$

is the general solution to the $h$-difference equation:

$$
\left(\Delta_{h}-\lambda_{r} I\right)^{m_{r}}\left(\Delta_{h}-\lambda_{n-1} I\right)^{m_{r-1}} \cdots\left(\Delta_{h}-\lambda_{1} I\right)^{m_{1}} x(t)=0 .
$$

Using connect (75), we can find the general solution

$$
\begin{aligned}
y(s) & =\sum_{j=1}^{r} \frac{q-1}{h}\left(\alpha_{j}(q-1)+1\right)^{\log _{q} s}\left[d_{j 0}+d_{j 1} h \log _{q} s+d_{j 2}\left(h \log _{q} s\right)^{2}+\cdots+d_{j m_{j}-1}\left(h \log _{q} s\right)^{m_{j}-1}\right] \\
& =\sum_{j=1}^{r}\left(\alpha_{j}(q-1)+1\right)^{\log _{q} s}\left[b_{j 0}+b_{j 1} \log _{q} s+b_{j 2}\left(\log _{q} s\right)^{2}+\cdots+b_{j m_{j}-1}\left(\log _{q} s\right)^{m_{j}-1}\right],
\end{aligned}
$$

of (73).

Corollary 1 is extended to the following result.

Corollary 3. Assume $\alpha_{j} \in \mathbb{C} \backslash\{-1 /(q-1)\}$ with $\mid \alpha_{j}(q-1)+$ $1 \mid \neq 1$ for $j \in\{1,2, \ldots, n\}$.

(i) If $\alpha_{j}(q-1)+1 \in(0,1) \cup(1, \infty)$ for $j \in\{1,2, \ldots, n\}$, then the nth-order quantum equation of Euler type (42) has Hyers-Ulam stability with minimum HUS constant

$$
K=\frac{1}{\prod_{j=1}^{n}\left|\alpha_{j}\right|}
$$

on $q^{\mathbb{Z}}$.

(ii) If $\quad \alpha_{j}(q-1)+1 \in(-\infty,-1) \cup(-1,0) \quad$ for $j \in\{1,2, \ldots, n\}$, then the nth-order quantum equation of Euler type (42) has Hyers-Ulam stability with minimum HUS constant

$$
K=\frac{(q-1)^{n}}{\prod_{j=1}^{n}\left|\alpha_{j}(q-1)+2\right|}
$$

on $q^{\mathbb{Z}}$.

Proof. Assume $\alpha_{j} \in \mathbb{C} \backslash\{-1 /(q-1)\}$ with $\left|\alpha_{j}(q-1)+1\right| \neq 1$ for $j \in\{1,2, \ldots, n\}$.

(i) If $\alpha_{j}(q-1)+1 \in(0,1) \cup(1, \infty)$ for $j \in\{1,2, \ldots, n\}$, then (42) has Hyers-Ulam stability with an HUS constant

$$
\prod_{j=1}^{n} K\left(q-1, \alpha_{j}\right)=\frac{1}{\prod_{j=1}^{n}\left|\alpha_{j}\right|}
$$

on $q^{\mathbb{Z}}$ from Theorem 3 .

We will prove that any HUS constant for (42) is greater than or equal to this constant. Let $\varepsilon>0$ be given. If we suppose that $0<K_{1}<1 / \prod_{j=1}^{n}\left|\alpha_{j}\right|$, and for any function $\psi$ satisfying

$\left|\left(s D_{q}-\alpha_{n} I\right)\left(s D_{q}-\alpha_{n-1} I\right) \cdots\left(s D_{q}-\alpha_{1} I\right) \psi(s)\right| \leq \varepsilon$, 
there exists a solution $y$ of (42) such that $|\psi(s)-y(s)| \leq K_{1} \varepsilon$ for all $s \in q^{\mathbb{Z}}$. Define

$$
\gamma_{1}(s):=\frac{\varepsilon}{\prod_{j=1}^{n}\left|\alpha_{j}\right|} .
$$

Then, $\gamma_{1}$ satisfies

$$
\begin{aligned}
& \left(s D_{q}-\alpha_{n} I\right)\left(s D_{q}-\alpha_{n-1} I\right) \cdots\left(s D_{q}-\alpha_{1} I\right) \gamma_{1}(s) \\
& =\left(-\alpha_{n}\right)\left(-\alpha_{n-1}\right) \cdots\left(-\alpha_{1}\right) \gamma_{1}(s) .
\end{aligned}
$$

This implies that

$$
\left|\left(s D_{q}-\alpha_{n} I\right)\left(s D_{q}-\alpha_{n-1} I\right) \cdots\left(s D_{q}-\alpha_{1} I\right) \gamma_{1}(s)\right|=\varepsilon,
$$

for all $s \in q^{\mathbb{Z}}$. Thus, by the assumption, we can find a solution $y_{1}$ of (42) such that $\left|\gamma_{1}(s)-y_{1}(s)\right| \leq K_{1} \varepsilon$ for all $s \in q^{\mathbb{Z}}$. By Lemma 3, we can rewrite $y_{1}$ as

$$
y_{1}(s)=\sum_{j=1}^{n} a_{j}\left(\alpha_{j}(q-1)+1\right)^{\log _{q} s}
$$

$$
\begin{aligned}
\text { or } y_{1}(s)= & \sum_{j=1}^{r}\left(\alpha_{j}(q-1)+1\right)^{\log _{q} s}\left[b_{j 0}+b_{j 1} \log _{q} s+b_{j 2}\left(\log _{q} s\right)^{2}\right. \\
& \left.+\cdots+b_{j m_{j}-1}\left(\log _{q} s\right)^{m_{j}-1}\right],
\end{aligned}
$$

where $a_{j}$ and $b_{j i}\left(i \in\left\{0,1,2, \ldots, m_{j}-1\right\}\right)$ are arbitrary constants so that we have

$$
\begin{aligned}
& \left|\gamma_{1}(s)-\sum_{j=1}^{n} a_{j}\left(\alpha_{j}(q-1)+1\right)^{\log _{q} s}\right|=\left|\gamma_{1}(s)-y_{1}(s)\right| \leq K_{1} \varepsilon \\
& \operatorname{or}\left|\gamma_{1}(s)-\sum_{j=1}^{r}\left(\alpha_{j}(q-1)+1\right)^{\log _{q} s}\left[b_{j 0}+b_{j 1} \log _{q} s+b_{j 2}\left(\log _{q} s\right)^{2}+\cdots+b_{j m_{j}-1}\left(\log _{q} s\right)^{m_{j}-1}\right]\right| \leq K_{1} \varepsilon .
\end{aligned}
$$

Since

$\lim _{s \rightarrow \infty}\left(\alpha_{j}(q-1)+1\right)^{\log _{q} s}=\infty, \quad$ if $\alpha_{j}(q-1)+1 \in(1, \infty)$ or $\lim _{s \rightarrow+0}\left(\alpha_{j}(q-1)+1\right)^{\log _{q} s}=\infty, \quad$ if $\alpha_{j}(q-1)+1 \in(0,1)$,

holds for all $j \in\{1,2, \ldots, n\}$, we conclude that $a_{j}=0$ for all $j \in\{1,2, \ldots, n\}$ and $b_{j i}=0$ for all $j \in\{1,2, \ldots, r\}$ and $i \in\left\{0,1,2, \ldots, m_{j}-1\right\}$. That is, $y_{1}(s) \equiv 0$. Hence, we see that

$$
K_{1} \varepsilon \geq\left|\gamma_{1}(s)-y_{1}(s)\right|=\left|\gamma_{1}(s)\right|=\frac{\varepsilon}{\prod_{j=1}^{n}\left|\alpha_{j}\right|>K_{1} \varepsilon .}
$$

This is a contradiction. Thus, $1 / \prod_{j=1}^{n}\left|\alpha_{j}\right|$ is the minimum HUS constant.

(ii) If $\alpha_{j}(q-1)+1 \in(-\infty,-1) \cup(-1,0)$ for $j \in\{1,2$, $\ldots, n\}$, then (42) has Hyers-Ulam stability with an HUS constant

$$
\prod_{j=1}^{n} K\left(q-1, \alpha_{j}\right)=\frac{(q-1)^{n}}{\prod_{j=1}^{n}\left|\alpha_{j}(q-1)+2\right|}
$$

on $q^{\mathbb{Z}}$ from Theorem 3 .

We will prove that any HUS constant for (42) is greater than or equal to this constant. Let $\varepsilon>0$ be given. If we suppose that $0<K_{2}<\left((q-1)^{n} / \prod_{j=1}^{n}\left|\alpha_{j}(q-1)+2\right|\right)$, and for any function $\psi$ satisfying

$\left|\left(s D_{q}-\alpha_{n} I\right)\left(s D_{q}-\alpha_{n-1} I\right) \cdots\left(s D_{q}-\alpha_{1} I\right) \psi(s)\right| \leq \varepsilon$,

there exists a solution $y$ of (42) such that $|\psi(s)-y(s)| \leq K_{2} \varepsilon$ for all $s \in q^{\mathbb{Z}}$. Define

$$
\gamma_{2}(s):=\frac{(q-1)^{n} \varepsilon}{\prod_{j=1}^{n}\left|\alpha_{j}(q-1)+2\right|(-1)^{\log _{q} s}}
$$

Since

$$
\left(s D_{q}-\alpha_{j} I\right) \gamma_{2}(s)=s \frac{(q-1)^{n} \varepsilon}{\prod_{j=1}^{n}\left|\alpha_{j}(q-1)+2\right|(-1)^{\log _{q} q s}-(-1)^{\log _{q} s} /(q-1) s-\alpha_{j} \gamma_{2}(s)=\left(-2 / q-1-\alpha_{j}\right) \gamma_{2}(s)},
$$


holds, we have

$$
\begin{aligned}
& \left(s D_{q}-\alpha_{n} I\right)\left(s D_{q}-\alpha_{n-1} I\right) \cdots\left(s D_{q}-\alpha_{1} I\right) \gamma_{1}(s) \\
& =\left(\frac{-2}{q-1}-\alpha_{n}\right)\left(\frac{-2}{q-1}-\alpha_{n-1}\right) \cdots\left(\frac{-2}{q-1}-\alpha_{1}\right) \gamma_{2}(s) .
\end{aligned}
$$

This implies that

$$
\left|\left(s D_{q}-\alpha_{n} I\right)\left(s D_{q}-\alpha_{n-1} I\right) \cdots\left(s D_{q}-\alpha_{1} I\right) \gamma_{2}(s)\right|=\varepsilon,
$$

for all $s \in q^{\mathbb{Z}}$. Thus, by the assumption, we can find a solution $y_{2}$ of (42) such that $\left|\gamma_{2}(s)-y_{2}(s)\right| \leq K_{2} \varepsilon$ for all $s \in q^{\mathbb{Z}}$. By Lemma 3, we can rewrite $y_{2}$ as

$$
\begin{aligned}
y_{2}(s)= & \sum_{j=1}^{n} c_{j}\left(\alpha_{j}(q-1)+1\right)^{\log _{q} s} \\
\text { or } y_{2}(s)= & \sum_{j=1}^{r}\left(\alpha_{j}(q-1)+1\right)^{\log _{q} s}\left[d_{j 0}+d_{j 1} \log _{q} s\right. \\
& \left.+d_{j 2}\left(\log _{q} s\right)^{2}+\cdots+d_{j m_{j}-1}\left(\log _{q} s\right)^{m_{j}-1}\right],
\end{aligned}
$$

where $c_{j}$ and $d_{j i}\left(i \in\left\{0,1,2, \ldots, m_{j}-1\right\}\right)$ are arbitrary constants so that we have

$$
\begin{aligned}
& \left|\gamma_{2}(s)-\sum_{j=1}^{n} c_{j}\left(\alpha_{j}(q-1)+1\right)^{\log _{q} s}\right|=\left|\gamma_{2}(s)-y_{2}(s)\right| \leq K_{2} \varepsilon \\
& \text { or }\left|\gamma_{2}(s)-\sum_{j=1}^{r}\left(\alpha_{j}(q-1)+1\right)^{\log _{q} s}\left[d_{j 0}+d_{j 1} \log _{q} s+d_{j 2}\left(\log _{q} s\right)^{2}+\cdots+d_{j m_{j}-1}\left(\log _{q} s\right)^{m_{j}-1}\right]\right| \leq K_{2} \varepsilon
\end{aligned}
$$

Since

$\lim _{s \longrightarrow \infty}\left|\left(\alpha_{j}(q-1)+1\right)^{\log _{q} s}\right|=\infty, \quad$ if $\alpha_{j}(q-1)+1 \in(-\infty,-1)$

or $\lim _{s \longrightarrow+0}\left|\left(\alpha_{j}(q-1)+1\right)^{\log _{q} s}\right|=\infty, \quad$ if $\alpha_{j}(q-1)+1 \in(-1,0)$,

holds for all $j \in\{1,2, \ldots, n\}$, we conclude that $c_{j}=0$ for all $j \in\{1,2, \ldots, n\}$ and $d_{j i}=0$ for all $j \in\{1,2, \ldots, r\}$ and $i \in\left\{0,1,2, \ldots, m_{j}-1\right\}$. That is, $y_{2}(s) \equiv 0$. Hence, we see that

$$
K_{2} \varepsilon \geq\left|\gamma_{2}(s)-y_{2}(s)\right|=\left|\gamma_{2}(s)\right|=\frac{(q-1)^{n} \varepsilon}{\prod_{j=1}^{n}\left|\alpha_{j}(q-1)+2\right|>K_{2} \varepsilon}
$$

This is a contradiction. Thus, $(q-1)^{n} / \prod_{j=1}^{n} \mid \alpha_{j}(q-1)+$ $2 \mid$ is the minimum HUS constant.

Using Corollary 3 and connect (75), we can obtain the following result.

Corollary 4. Assume $\lambda_{j} \in \mathbb{C} \backslash\{-1 / h\}$ with $\left|\lambda_{j} h+1\right| \neq 1$ for $j \in\{1,2, \ldots, n\}$.

(i) If $\lambda_{j} h+1 \in(0,1) \cup(1, \infty)$ for $j \in\{1,2, \ldots, n\}$, then the nth-order h-difference equation with constant coefficients (70) has Hyers-Ulam stability with minimum HUS constant

$$
K=\frac{1}{\prod_{j=1}^{n}\left|\lambda_{j}\right|}
$$

on $q^{\mathbb{Z}}$.

(ii) If $\lambda_{j} h+1 \in(-\infty,-1) \cup(-1,0)$ for $j \in\{1,2, \ldots, n\}$, then the nth-order $h$-difference equation with constant coefficients (70) has Hyers-Ulam stability with minimum HUS constant

$$
K=\frac{h^{n}}{\prod_{j=1}^{n}\left|\lambda_{j} h+2\right|}
$$

on $q^{\mathbb{Z}}$.

\section{Perturbed Quantum Equations of Euler Type}

In this section, we consider the $n$ th-order perturbed quantum equation of the Euler type:

$$
\begin{array}{r}
\left(s D_{q}-\alpha_{n} I\right)\left(s D_{q}-\alpha_{n-1} I\right) \cdots\left(s D_{q}-\alpha_{1} I\right) y(s)=g(s), \\
D_{q} y(s):=\frac{y(q s)-y(s)}{(q-1) s},
\end{array}
$$

where $g: q^{\mathbb{Z}} \longrightarrow \mathbb{C}$ is a perturbation. By using Theorem 3 , we obtain the following result. 
Theorem 4. If $\alpha_{j} \in \mathbb{C} \backslash\{-1 /(q-1)\}$ with $\left|\alpha_{j}(q-1)+1\right| \neq 1$ for $j \in\{1,2, \ldots, n\}$, then the higher-order perturbed quantum equation of Euler type (106) has Hyers-Ulam stability with an HUS constant of

$$
\prod_{j=1}^{n} K\left(q-1, \alpha_{j}\right)=\frac{(q-1)^{n}}{\prod_{j=1}^{n}|1-| \alpha_{j}(q-1)+1||}
$$

on $q^{\mathbb{Z}}$, where $K(\cdot, \cdot)$ is given in (13).

Proof. Let $w: q^{\mathbb{Z}} \longrightarrow \mathbb{C}$ be a solution of (106), and let $\varepsilon>0$ be given. Suppose that $\psi: q^{\mathbb{Z}} \longrightarrow \mathbb{C}$ satisfies

$$
\begin{array}{r}
\left(s D_{q}-\alpha_{n} I\right)\left(s D_{q}-\alpha_{n-1} I\right) \cdots\left(s D_{q}-\alpha_{1} I\right) \psi(s)-g(s) \mid \leq \varepsilon, \\
s \in q^{\mathbb{Z}} .
\end{array}
$$

Since $w$ is a solution of (106), we have

$\left|\left(s D_{q}-\alpha_{n} I\right)\left(s D_{q}-\alpha_{n-1} I\right) \cdots\left(s D_{q}-\alpha_{1} I\right)(\psi(s)-w(s))\right| \leq \varepsilon$.

Using Theorem 3, we can find a solution $z: q^{\mathbb{Z}} \longrightarrow \mathbb{C}$ of (42) such that

$$
|(\psi(s)-w(s))-z(s)| \leq \frac{(q-1)^{n} \varepsilon}{\prod_{j=1}^{n}|1-| \alpha_{j}(q-1)+1||}, \quad s \in q^{\mathbb{Z}} .
$$

Note here that $w(s)+z(s)$ is a solution of (106) because

$$
\begin{aligned}
& \left(s D_{q}-\alpha_{n} I\right)\left(s D_{q}-\alpha_{n-1} I\right) \cdots\left(s D_{q}-\alpha_{1} I\right)(w(s)+z(s)) \\
& =g(s)+0=g(s),
\end{aligned}
$$

holds. This says that (106) has Hyers-Ulam stability.

Using Theorem 4 and connect (75) with $f(t)=g\left(q^{t / h}\right)$, we can establish the following result.

Theorem 5. If $\lambda_{j} \in \mathbb{C} \backslash\{-1 / h\}$ with $\left|\lambda_{j} h+1\right| \neq 1$ for $j \in\{1,2, \ldots, n\}$, then the higher-order perturbed $h$-difference equation with constant coefficients

$$
\left(\Delta_{h}-\lambda_{n} I\right)\left(\Delta_{h}-\lambda_{n-1} I\right) \cdots\left(\Delta_{h}-\lambda_{1} I\right) x(t)=f(t),
$$

has Hyers-Ulam stability with an HUS constant of

$$
\prod_{j=1}^{n} K\left(h, \lambda_{j}\right)=\frac{h^{n}}{\prod_{j=1}^{n}|1-| \lambda_{j} h+1||}
$$

on $h \mathbb{Z}$, where $K(\cdot, \cdot)$ is given in (13).

\section{Conclusion}

New results connecting $h$-difference equations with complex constant coefficients and $q$-difference equations of the Euler type are presented, for equations of all integer orders. For equation orders greater than two, the $h$-difference results extend first-order and second-order results found in the literature, and the Euler-type $q$-difference results are completely novel for any order. In many cases, the best HUS constant is found. The key idea introduced here is establishing results for one type of equation and then converting those results over to the other through a change of the variable.

\section{Data Availability}

No data were used to support this study.

\section{Conflicts of Interest}

The authors declare that they have no conflicts of interest.

\section{Acknowledgments}

Professor Onitsuka was supported by JSPS KAKENHI Grant no. JP20K03668. This work was also supported by the Research Institute for Mathematical Sciences, an International Joint Usage/Research Center located in Kyoto University.

\section{References}

[1] V. Kac and P. Cheung, Quantum Calculus, Springer, New York, NY, USA, 2001.

[2] D. R. Anderson and M. Onitsuka, "Best constant for Hyers-Ulam stability of second-order $h$-difference equations with constant coefficients," Results in Mathematics, vol. 74, no. 4, p. 151, 2019.

[3] M. Onitsuka, "Hyers-Ulam stability of second-order nonhomogeneous linear difference equations with a constant step size," Journal of Computational Analysis and Applications, vol. 28, no. 1, pp. 152-165, 2020.

[4] C. Buşe, V. Lupulescu, and D. O’Regan, "Hyers-Ulam stability for equations with differences and differential equations with time-dependent and periodic coefficients," Proceedings of the Royal Society of Edinburgh: Section A Mathematics, pp. 1-14, 2019.

[5] Y. Shen and Y. Li, "The $z$-transform method for the Ulam stability of linear difference equations with constant coefficients," Advances in Difference Equations, vol. 2018, no. 1, p. 396, 2018.

[6] B. Xu and J. Brzdęk, "Hyers-Ulam stability of a system of first order linear recurrences with constant coefficients," Discrete Dynamics in Nature and Society, vol. 2015, Article ID 269356, 5 pages, 2015.

[7] S. Elaydi, An Introduction to Difference Equations, Springer, New York, NY, USA, Undergraduate Texts in Mathematics, 3rd edition, 2005. 